\title{
A new theoretical approach for treatment of GIXD maps
}

\author{
Grigory Prutskov ${ }^{1}$, Mikhail Chuev ${ }^{2}$, Sergey Yakunin $^{2}$, Natalia Novikova ${ }^{2}$, Alexander Rogachev ${ }^{2}$, Oleg Konovalov ${ }^{3}$ \\ ${ }^{1}$ National Research Centre "Kurchatov Institute", Moscow, Russian Federation, ${ }^{2}$ National Research Centre, Moscow, Russian \\ Federation, ${ }^{3}$ European Synchrotron Radiation Facility, Grenoble, France \\ E-mail: prutskov@zoho.com
}

The development of new approaches to structural diagnostics of bioorganic molecular films is indispensable key to success in advanced nanotechnologies. Surface-sensitive X-ray techniques, such as grazing incidence diffraction (GIXD), provide the fullest insight into the structural parameters of nanosized materials and processes that flow within them. Highly intensive Xray sources - synchrotrons, offered a real opportunity to implement GIXD measurements on weakly scattering systems and brought the diagnostics of bioorganic films to a fundamentally new level.

Commonly used methods for calculation GIXD data of Langmuir films are qualitative in nature or based on the powder diffraction approximations [1] which do not take into account specific interaction between ordered molecules and radiation under total external reflection conditions.

The elaborated theoretical approach takes into consideration strongly limited penetration depth, random orientation of Langmuir film clusters, distribution of the monolayer parameters in the film and instrumental function. For quantitative analysis of GIXD scattering maps new software has been developed based on this approach which allows to fitting 2D experimental maps and provides detailed information about radiation damage and real structure of 2D molecular nanosystems.

The crucial point of this algorithm is decomposition of incidence wave by two components according to distorted wave approximation [2], whereby one of them responds for taking into account total external reflection conditions and the another component examines scattering on the periodic system. Description of the random clusters orientation is archived by intensity integration in the small angular range near Bragg peaks where intensities of the scattered radiation is not equal to zero.

Above approach assures the determination not only average 2D lattice constants, tilt and rotation angles of the constituting monolayer molecules, but also standard deviation of these parameters from the average values and size of film clusters.

[1] Kjaer K. (1994), Physica B 198, 100-109.

[2] Vineyard. J.H., (1982), Phys. Rev. B 26, 4146.

a

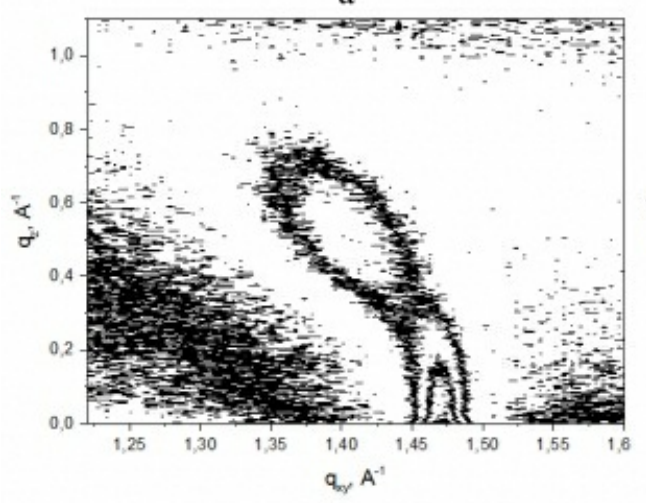

b

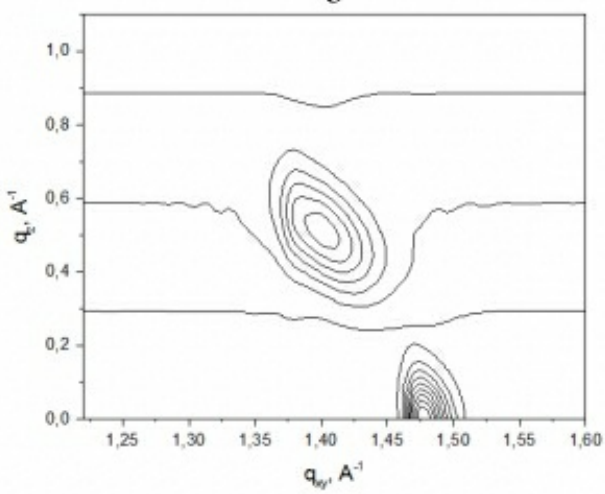

Experimental (a) and simulated (b) GID maps for Langmuir monolayer of DPPC

Keywords: Langmuir films, grazing incidence diffraction, synchrotron radiation 